\title{
RANDOMISED DOUBLE-BLIND STUDY OF INTRATHECAL BUPIVACAINE-MORPHINE VERSUS SYSTEMIC MORPHINE ANALGESIA FOR MAJOR ABDOMINAL SURGERY IN A RESOURCE POOR SETTING
}

\author{
Shreyasi Ray ${ }^{1}$, Jyotirmay Kirtania ${ }^{2}$
}

${ }^{1}$ Assistant Professor, Department of Anaesthesiology, ESI-PGIMSR and ESIC Medical College, Joka, Kolkata, India. ${ }^{2}$ Associate Professor, Department of Anaesthesiology, ESI-PGIMSR and ESIC Medical College, Joka, Kolkata, India.

\section{ABSTRACT}

\section{BACKGROUND}

Failure of adequate postoperative analgesia, defined as Numerical Rating Scale (NRS) pain score $>3$ after major abdominal surgery have been reported to be $30 \%$ to $50 \%$ in a large number of studies. Effective yet inexpensive technique of anaesthesia and postoperative analgesia for major abdominal surgery seemed to be a hitherto elusive entity.

\section{MATERIALS AND METHODS}

In this randomised, double-blinded study involving ninety-two participants, we investigated the clinical efficacy and safety of morphine plus bupivacaine administered intrathecally versus systemic morphine for pain control in the first 24h postoperative period after major abdominal surgery under relaxant general anaesthesia in a resource-poor setting.

\section{RESULTS}

Our experimental protocol had significant ARR of 0.3043 (95\% CI: 0.1554 to 0.4497 ) at $12 \mathrm{~h}$ and ARR of 0.3261 (95\% CI: 0.1579 to 0.4764 ) at $24 \mathrm{~h}$ in the failure of adequate postoperative analgesia (NRS pain score $>3$ ) with an NNT of 3 . There were no significant differences in intraoperative vasopressor consumption, the postoperative OASS scores, first 24h urine output, incidence of postoperative nausea and vomiting and Michigan Awareness Classification Instrument Class. The experimental group experienced higher incidence of mild pruritus. The incidence of serious adverse events was nil.

\section{CONCLUSION}

In patients undergoing major abdominal surgery under relaxant general anaesthesia in a simulated resource poor setting, intrathecal analgesia with bupivacaine plus morphine is safe and reduces the incidence of failure of adequate analgesia in the first $24 \mathrm{~h}$ postoperative period.

\section{KEYWORDS}

Intrathecal Analgesia, Major Abdominal Surgery, Resource Poor Setting.

HOW TO CITE THIS ARTICLE: Ray S, Kirtania J. Randomised double-blind study of intrathecal bupivacaine-morphine versus systemic morphine analgesia for major abdominal surgery in a resource poor setting. J. Evolution Med. Dent. Sci. 2017;6(75):53455352, DOI: $10.14260 /$ Jemds/2017/1161

\section{BACKGROUND}

Postoperative pain control is one of the cornerstones of good quality anaesthesia care. Various modalities of postoperative analgesia are available. They range from highly effective, but highly resource-intensive and expensive techniques (e.g. patient controlled intravenous analgesia- PCA, patient controlled epidural analgesia- PCEA, nurse controlled intravenous analgesia- NCA and continuous subcutaneous infusion), to relatively less effective but less resourceintensive and cheaper techniques (e.g. intermittent subcutaneous or intramuscular morphine injections).[1]

Intrathecal morphine alone in doses of $0.05 \mathrm{mg}$ to $1 \mathrm{mg}$ have been reported to be safe and effective for postoperative analgesia in patients undergoing cardiac and vascular surgery under general anaesthesia. ${ }^{[2,3,4,5]}$

Financial or Other, Competing Interest: None.

Submission 28-08-2017, Peer Review 10-09-2017,

Acceptance 12-09-2017, Published 18-09-2017.

Corresponding Author:

Jyotirmay Kirtania,

\#9/1/2, BC Roy Path,

Shyamnagar, North 24 Parganas,

West Bengal, India.

E-mail: jyoti.kirtania@rediffmail.com

DOI: $10.14260 /$ jemds $/ 2017 / 1161$

\section{(c) $(\mathrm{P})(-$}

Intrathecal morphine in doses of $0.05 \mathrm{mg}$ to $0.3 \mathrm{mg}$ have been safely used as adjunct to intrathecal local anaesthetic agents for postoperative analgesia after caesarean section, and orthopaedic surgery under spinal anaesthesia.[6,7]

Wongyingsinn et al have shown in a randomised controlled trial that spinal analgesia with morphine combined with bupivacaine for laparoscopic colonic resection provide better analgesia and decreases postoperative opioid requirement than those treated with intravenous opioid PCA.[8] However, the efficacy and safety of intrathecal morphine plus bupivacaine combined with general anaesthesia for major abdominal surgery in a resource poor setting was not studied till date.

In this study, we investigated the clinical efficacy and safety of morphine plus bupivacaine administered intrathecally versus systemic morphine for pain control in the first 24 hours postoperative period after major abdominal surgery under general anaesthesia in a resource-poor setting.

\section{MATERIALS AND METHODS \\ Subjects}

This prospective, double-blinded, parallel-arm, randomised study was approved by the Institute Ethics Committee of the Medical College, Kolkata, India and registered with the Clinical Trials Registry of India (CTRI) with Clinical Trial 
registration no. CTRI/2014/06/004683. Male and female patients of ASA physical status 1, 2 and 3, between 18 and 60 years of age undergoing major abdominal surgery under general anaesthesia were included in the study. Patients having absolute contraindications for spinal anaesthesia, systemic or intrathecal morphine, pregnancy, advanced cardiovascular disease, cerebrovascular disease, dementia, respiratory disease, renal disease or liver disease with functional class worse than NYHA grade 2, circulatory shock, ASA physical status 4 or worse were excluded from the study. Figure 1 shows the study flow following Consolidated Standards of Reporting Trials guidelines. The study was conducted between January 2013 and December 2014.

\section{Study Design}

This study was designed to assess the clinical efficacy of morphine plus bupivacaine administered intrathecally versus systemic morphine for pain control in the first 24 hours postoperative period after major abdominal surgery under general anaesthesia in a resource-poor setting. Ninety-two consenting patients undergoing major abdominal surgery under relaxant general anaesthesia were randomised into two equal groups, to receive either intrathecal $0.25 \%$ isobaric bupivacaine $4 \mathrm{~mL}$ plus morphine $0.75 \mathrm{mg}(0.05 \mathrm{~mL})$ or receive intrathecal $0.9 \% \mathrm{NaCl}(4.05 \mathrm{~mL})$ plus intravenous morphine $0.2 \mathrm{mg} / \mathrm{kg}$ at the beginning of surgery plus subcutaneous morphine $0.1 \mathrm{mg} / \mathrm{kg}$ at the end of surgery. In the intrathecal analgesia group, general anaesthesia was maintained with intravenous midazolam $0.04 \mathrm{mg} / \mathrm{kg} / \mathrm{hour}$ with controlled ventilation with $50 \%$ oxygen in air mixture, on a low-cost electric piston driven ventilator (Sur Ventilator Mark V, Sur Electrical Co. Pvt. Ltd., Kolkata, India), without the use of any anaesthetic gases or volatile agents from any anaesthesia workstation. In the systemic analgesia group, general anaesthesia was maintained on controlled ventilation with isoflurane $1.5 \%$ (dial setting) in $50 \%$ oxygen with $50 \%$ nitrous oxide mixture on anaesthesia workstation (Penlon Prima SP102 with integrated AV800 Ventilator, Sigma-Delta Vaporizer and A100 Absorber, Penlon Ltd., UK).

\section{Randomisation}

Eligible patients underwent randomisation after providing written informed consent. The random sequence of allocation code (Intrathecal analgesia group or systemic analgesia group) was obtained from a random number table of integers. This random number table of integers was constructed using a computer generated random number function in LibreOffice Calc version 5.0.3.2.

Randomised and blinded allocation of patients to the study drugs was achieved by assigning concealed random number codes to patients at the time of enrolment. Labels indicating intrathecal analgesia group or systemic analgesia group were sealed in opaque, numbered envelopes. The concealed randomised allocation codes (patient's group assignment) was known only to the principal investigator and the anaesthesia care givers, but not to the postoperative assessors or the patients or the statistician.

\section{Calculation of Sample Size}

This was a prospective study of independent experimental subjects and controls with one control per experimental subject. Prior data indicate that the failure rate of adequate analgesia among controls is approximately $0.3(30 \%) .[1]$ If the true failure rate for experimental subjects is at least 0.05 $(5 \%)$, i.e. $25 \%$ relative risk reduction of failure of adequate analgesia in the experimental group, we needed to study at least forty six experimental subjects and forty six control subjects to be able to reject the null hypothesis that the failure rates for experimental and control subjects are equal with probability (power) 0.9 (90\%). The Type I error probability associated with this test of this null hypothesis is 0.05 (5\%). Uncorrected chi-square statistic was used to evaluate this null hypothesis. The sample size and power was calculated using the "PS Power and Sample Size Calculations" software Version 3.0, January 2009 Copyright $($ 1997-2009 by William D. Dupont and Walton D. Plummer.

\section{Outcome Measures}

1. The failure rate of adequate pain control (proportion of patients with pain intensity on NRS of more than $3 / 10$ ) at 30 minutes, at 12 hours and at 24 hours after tracheal extubation in the two groups of patients.

2. Pain score at 30 minutes, 12 hours and 24 hours following tracheal extubation in the two groups of patients. Pain score was measured on Numerical Rating Scale (NRS) of 0 to 10 , where ' 0 ' is no pain and ' 10 ' is the worst possible pain.

3. Total morphine consumption in the first 24 hours after tracheal extubation in the two groups of patients.

4. Observer Assessment of Sedation Scale (OASS) score ${ }^{[9]}$ at 30 minutes, 12 hours and 24 hours following tracheal extubation in the two groups of patients. OASS Score measured $[9]$ as: Responds readily to name spoken in normal tone (Alert) $=5$; Lethargic response to name spoken in normal tone (Light sedation) $=4$; Responds only after name is called loudly and/or repeatedly (Moderate sedation) $=3$; Responds only after mild prodding or shaking (Moderate sedation) $=2$; Does not respond to mild prodding or shaking (Deep sedation) $=$ 1.

5. Incidence of intraoperative awareness in the two groups of patients. Intraoperative awareness was assessed with the use of a modified Brice questionnaire. Patients were evaluated at 30 minutes after extubation and at 24 hours after extubation. Patients reporting memories of the period between 'going to sleep' and 'waking up' at any of the above two interviews were assessed by a different evaluator, who asked additional structured questions. Interviewers blinded to the study conducted the modified Brice interview,[10] which was defined by the following questions: (a) What was the last thing you remember before going to sleep?, (b) What is the first thing you remember after waking up?, (c) Do you remember anything between going to sleep and waking up?, (d) Did you dream during your procedure? and (e) What was the worst thing about your operation? Based on the data obtained from these interviews any reports suggestive of awareness were assessed and classified by assessors blinded to the study conditions. Awareness reports were classified as: (1) No awareness or awareness of something with a high probability of occurring in the immediate preoperative or postoperative period, (2) Possible awareness: patient 
unable to recall any event definitively indicative of awareness and (3) Definite awareness in which events are confirmed or have a high likelihood of occurring in the intraoperative period. Events of possible or definite awareness were assigned to one of the categories (Class 1 to Class 5) of the Michigan Awareness Classification Instrument. The patients with nil intraoperative memory were classified as Class 0 . Michigan Awareness Classification Instrument ${ }^{[0]}$ : Class 0 : No awareness; Class 1: Isolated auditory perceptions; Class 2: Tactile perceptions (e.g. surgical manipulation or endotracheal tube); Class 3: Pain; Class 4: Paralysis (e.g. feeling one cannot move, speak or breathe); Class 5: Paralysis and pain.

6. Urine output within the first 24 hours following the induction of anaesthesia in the two study groups.

7. Total intraoperative mephentermine (vasopressor) consumption in the intraoperative period in the two study groups.

8. The incidence of sustained hypotension (systolic blood pressure less than $90 \mathrm{mmHg}$ for more than 10 minutes), sustained bradycardia (heart rate less than 50 per minute for more than 3 minutes) and sustained severe tachycardia (heart rate more than 180 minus age per minute for more than 3 minutes) during the intraoperative period.

9. The incidences of postoperative adverse events like respiratory depression (respiratory rate of less than 10 breaths per minute), hypoxia (SpO2 < 92\%), hypotension (systolic BP $<90 \mathrm{mmHg}$ ), nausea and vomiting, mild pruritus, severe pruritus (requiring treatment), postoperative headache and any other serious adverse events within the first 48 hours after completion of the surgery.

All the study participants received premedication of oral tab. diazepam $5 \mathrm{mg}$ plus oral tab. pantoprazole $40 \mathrm{mg}$ at night before surgery and repeated at $6 \mathrm{AM}$ on the morning of surgery. In the preoperative area, two 18G intravenous access were secured in two large peripheral veins of the hand. In one cannula, maintenance intravenous fluid infusion @ 60 to 85 $\mathrm{mL} / \mathrm{h}$ was started with Inj. $0.45 \% \mathrm{NaCl}+10 \mathrm{mmol} / \mathrm{L} \mathrm{KCl}$ (prepared by adding $5 \mathrm{mmol} \mathrm{Inj}$. $\mathrm{KCl}$ to $500 \mathrm{~mL}$ bottle of $0.45 \% \mathrm{NaCl}$ ). The other cannula was used for infusion of colloids or blood products as required. In the operating room, each patient received intravenous premedication of inj. dexamethasone $0.8 \mathrm{mg} / \mathrm{kg}$ for prevention of postoperative nausea and vomiting before induction of anaesthesia. Intravenous prophylactic antibiotics for surgical site infection was administered as per local protocol for the particular surgery. General anaesthesia with tracheal intubation and controlled ventilation was induced with sleep dose of inj. thiopentone ( 4 to $5 \mathrm{mg} / \mathrm{kg}$ ) IV over 20 seconds and inj. suxamethonium $1.5 \mathrm{mg} / \mathrm{kg}$ IV over 2 seconds. Inj. paracetamol $20 \mathrm{mg} / \mathrm{kg}$ (subject to a maximum of $1 \mathrm{~g}$ ) intravenous infusion over 15 minutes was given to all study participants soon after induction of general anaesthesia.

In the intrathecal analgesia group, general anaesthesia was maintained with intravenous midazolam 0.04 $\mathrm{mg} / \mathrm{kg} /$ hour (or $0.02 \mathrm{mg} / \mathrm{kg} /$ hour for patients above 50 years age) with volume controlled ventilation with $50 \%$ oxygen in air mixture, on a low-cost electric-piston driven ventilator (Sur Ventilator Mark V, Sur Electrical Co. Pvt. Ltd., Kolkata, India) without the use of any anaesthesia workstation, nitrous oxide or volatile agents. Minute ventilation of 120 to $200 \mathrm{~mL} / \mathrm{kg} / \mathrm{min}$ was targeted to maintain ETCO2 between 32 and $40 \mathrm{mmHg}$.

In the systemic analgesia group, general anaesthesia was maintained on controlled ventilation with isoflurane $1.5 \%$ (dial setting) in $50 \%$ oxygen with $50 \%$ nitrous oxide mixture on anaesthesia workstation (Penlon Prima SP102 with integrated AV800 Ventilator, Sigma-Delta Vaporizer and A100 Absorber, Penlon Ltd., UK).

In both the groups, surgical muscle relaxation was maintained with vecuronium $70 \mathrm{mcg} / \mathrm{kg}$ intravenous loading followed by $20 \mathrm{mcg} / \mathrm{kg}$ intravenous bolus every 30 minutes or as required. Urinary bladder was catheterised aseptically and the open end of catheter attached to an urobag for monitoring of urine output.

All the study participants were monitored intraoperatively by 5 lead ECG, Sp02, automated NIBP, ETCO2, core temperature and hourly urine output.

Ninety-two study participants were randomised into two equal groups, to receive either intrathecal $0.25 \%$ isobaric bupivacaine $4 \mathrm{~mL}$ plus morphine $0.75 \mathrm{mg}(0.05 \mathrm{~mL})$ or receive intrathecal $0.9 \% \mathrm{NaCl}(4.05 \mathrm{~mL})$ plus intravenous morphine $0.2 \mathrm{mg} / \mathrm{kg}$ at the beginning of surgery plus subcutaneous morphine $0.1 \mathrm{mg} / \mathrm{kg}$ at the end of surgery.

Technique of intrathecal injection: Before the induction of general anaesthesia, subarachnoid block under strict aseptic position was performed in each patient at L3/4 or L4/5 space using a $9 \mathrm{~cm}$ 26G Quincke needle with the code allocated solution injected @ $0.2 \mathrm{~mL} /$ second.

\section{The Study Drugs were allocated as follows}

- For the Experimental Group:

a. Intrathecal Injection: Sterile, preservative free $0.25 \%$ bupivacaine $4 \mathrm{~mL}+$ preservative free morphine 0.75 $\mathrm{mg}(0.05 \mathrm{~mL})$, total volume approximately $4.05 \mathrm{~mL}$, aseptically prepared. The measured amount of morphine $(0.75 \mathrm{mg}=5$ small divisions $)$ was added from a sterile tuberculin syringe $(1 \mathrm{~mL}=100$ small divisions).

b. Intravenous injection of $0.9 \% \mathrm{NaCl}(1 \mathrm{~mL})$ was given immediately before the induction of general anaesthesia.

c. Subcutaneous injection of $0.9 \% \mathrm{NaCl}(0.5 \mathrm{~mL})$ was given at the start of abdominal wound closure using a $26 \mathrm{G} \times 0.5$ " needle.

\section{- For the Control Group:}

a. Intrathecal injection: Sterile, preservative free $0.9 \%$ $\mathrm{NaCl}(4.05 \mathrm{~mL})$.

b. Intravenous injection of morphine sulphate 0.2 $\mathrm{mg} / \mathrm{kg}$, diluted with $0.9 \% \mathrm{NaCl}$ to make $1 \mathrm{~mL}$, was given immediately before the induction of general anaesthesia.

c. Subcutaneous injection morphine sulphate 0.1 $\mathrm{mg} / \mathrm{kg}$, diluted with $0.9 \% \mathrm{NaCl}$ to make $0.5 \mathrm{~mL}$ was given at the start of abdominal wound closure using a $26 \mathrm{G} \times 0.5$ " needle.

Blood loss replacement during surgery was done with inj. succinylated polygeline $4 \%$ in normal saline, equal to the estimated volume of blood loss. Blood loss beyond the 
estimated maximum allowable blood loss or in presence of clinical signs of acute anaemic hypoxia, was treated by intraoperative packed RBC transfusion as per WHO transfusion guidelines. The postoperative target haemoglobin was between 9 and $10 \mathrm{~g} / \mathrm{dL}$.

If there was intraoperative hypotension (SBP $<90$ $\mathrm{mmHg}$ ) despite adequate fluid replacement, it was corrected with incremental intravenous bolus of inj. mephentermine 6 mg. Patients remaining hypotensive even after $60 \mathrm{mg}$ of intravenous inj. mephentermine, were treated with infusion of inj. noradrenaline $(100 \mathrm{mcg} / \mathrm{mL}$ dilution $)$ starting at 0.1 $\mathrm{mcg}^{*} \mathrm{~kg}^{-1} * \mathrm{~min}^{-1}$.

Temperature Management: Before the induction of anaesthesia, all patients were pre-warmed for 5 minutes with a blower-fan type convective room heater (set to 750 watts output) placed 3 feet away from the patient. The patients were kept warm by insulating both lower limbs, upper limbs, chest and head-neck region with clean PVC sheets immediately after completion of intrathecal injection and urinary catheterisation. Any inadvertent hypothermia detected in the immediate postoperative period was corrected in the recovery area with a convective room heater with blower-fan (set to 750 watts output) placed 3 feet away from the patient (with additional surface temperature monitoring) till the patient was normothermic (core temperature between 36.8 and $37.2^{\circ} \mathrm{C}$ ).

Near the end of surgery (after start of abdominal wound closure), any further boluses of muscle relaxants were withheld. Intravenous inj. ondansetron $0.4 \mathrm{mg} / \mathrm{kg}$ was given to all patients for prophylaxis of postoperative nausea and vomiting. At the end of the surgery, neuromuscular blockade was reversed with intravenous inj. neostigmine $50 \mathrm{mcg} / \mathrm{kg}$ IV with inj. glycopyrrolate $10 \mathrm{mcg} / \mathrm{kg}$. Tracheal extubation was done when patient was able to do sustained tongue protrusion and sustained head lifting for more than 5 secs on verbal command.

Patients were sent to the postoperative ward on satisfying the recovery criteria: OASS score $>3$; able to do sustained ( $>5$ secs) head lift; respiratory rate between 12 and 30 breaths/min; able to cough and breathe deeply; SpO2 $>97 \%$ (on $4 \mathrm{~L} / \mathrm{min}$ supplemental oxygen by face mask); SBP $>100 \mathrm{mmHg}$; surgical wound pain score less than $3 / 10$ on NRS; and core temperature between 36.8 and $37.2^{\circ} \mathrm{C}$.

In the postoperative ward, patient received oxygen supplementation initially for at least 24 hours after completion of surgery. Initial postoperative care included intravenous fluids, antibiotics, analgesia, stress ulcer prophylaxis, thromboprophylaxis, nutritional support, treatment of co-existing diseases and other supportive care. Postoperative routine monitoring during the first 24 hours included continuous pulse oximetry, hourly monitoring of the level of consciousness, respiratory rate, automated NIBP measurement, temperature and hourly urine output.

\section{All Patients in both the Groups Received Postoperative} Analgesia as per the following Plan

- Inj. paracetamol $15 \mathrm{mg} / \mathrm{kg}$ intravenous infusion every 6 hours subject to a maximum of $4 \mathrm{~g}$ in 24 hours.

- $\quad$ Rescue analgesia with S/C inj. morphine $0.1 \mathrm{mg} / \mathrm{kg}$ (maximum of 6 doses in 24 hours), whenever the patient complains of surgical wound pain with an intensity of more than $3 / 10$ on NRS.
- No other sedative agents, opioid or analgesics were given to any patient in either group.

\section{Statistical Analysis}

Continuous variables were tested for normality of distribution using the Shapiro-Wilk normality test. Continuous data variables having non-normal distribution (nonparametric variables) were compared with the MannWhitney U (Wilcoxon Rank Sum) test and those with normal distribution were compared with Student's ' $t$ ' test. Categorical data (sex and ASA physical status) and proportions (failure rate of postoperative analgesia and complication rates) were analysed using the Pearson chisquare test or Fisher's Exact Test. A value of $\mathrm{P}<0.05$ was considered statistically significant. Data analysis was performed using the $\mathrm{R}$ Commander statistical package version 2.3.2 on base $\mathrm{R}$ version 3.2.3 (www.r-project.org) on 32 bit Linux. The risk statistics (absolute risk reduction [ARR], number needed to treat [NNT], relative risk reduction [RRR]) and the 95\% CI for the difference between two proportions was calculated using a spreadsheet utility based on the method proposed by Newcombe.[11]

\section{RESULTS}

Applying Shapiro-Wilk normality test, it was observed that all except one continuous variable had non-normal distribution. So all the continuous variables were expressed as median and interquartile range (IQR) and group medians were compared with Mann-Whitney U (Wilcoxon Rank Sum) test. Demographic characteristics of the two groups of patients are shown in Table 1. The intrathecal analgesia group had significantly lower pain intensity (NRS) at 30 minutes, 12 hours and 24 hours after tracheal extubation (Table 2). However, none of the patients in any of the groups had pain score of more than 3 (NRS) at 30 minutes following tracheal extubation. So there the failure rate of adequate analgesia in the immediate postoperative period in both the groups was nil (Table 3). The total morphine consumption in the first 24 hours period after extubation was lower in the intrathecal analgesia group (Table 2).

The failure rate of adequate postoperative analgesia at 12 hours and 24 hours following tracheal extubation (Proportion of patients with pain score of more than 3 on NRS) was significantly lower in the intrathecal analgesia group (Table 3).

There was no significant difference between the two groups in respect of OASS score at 30 minutes, 12 hours and 24 hours after tracheal extubation, total mephentermine (vasopressor) consumption in the intraoperative period and urine output in the first 24 hours following induction of anaesthesia (Table 2). Also, there was no significant difference in the incidence of postoperative nausea and vomiting, postoperative severe pruritus (requiring treatment) and Michigan Awareness Classification Instrument Class between the two groups. However, the incidence of mild pruritus was significantly higher in the intrathecal analgesia group (Table 4). None of the patients in any of the groups had any serious adverse events during the study period. 


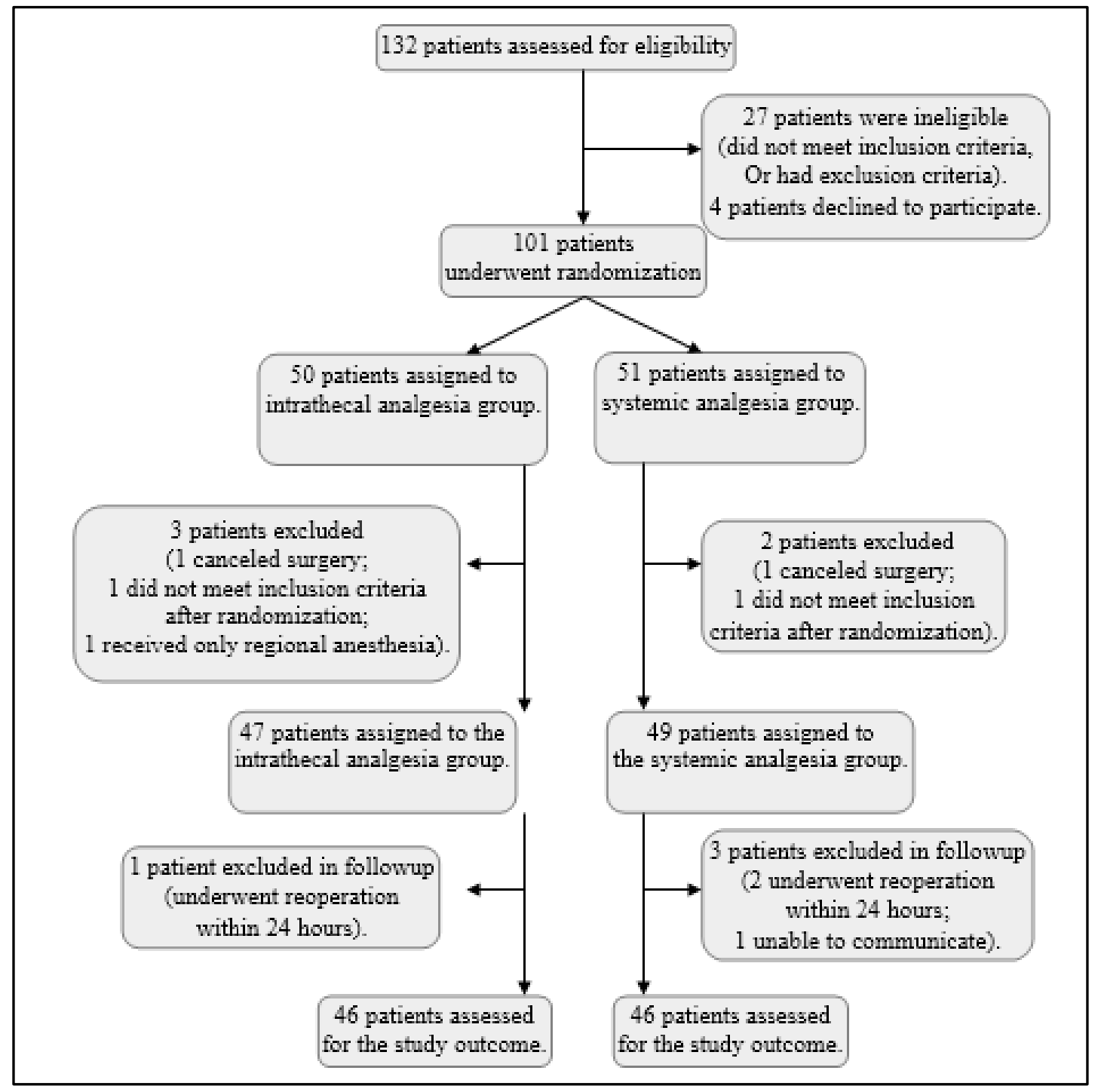

Figure 1. Study Flow following Consolidated Standards of Reporting Trials Guidelines

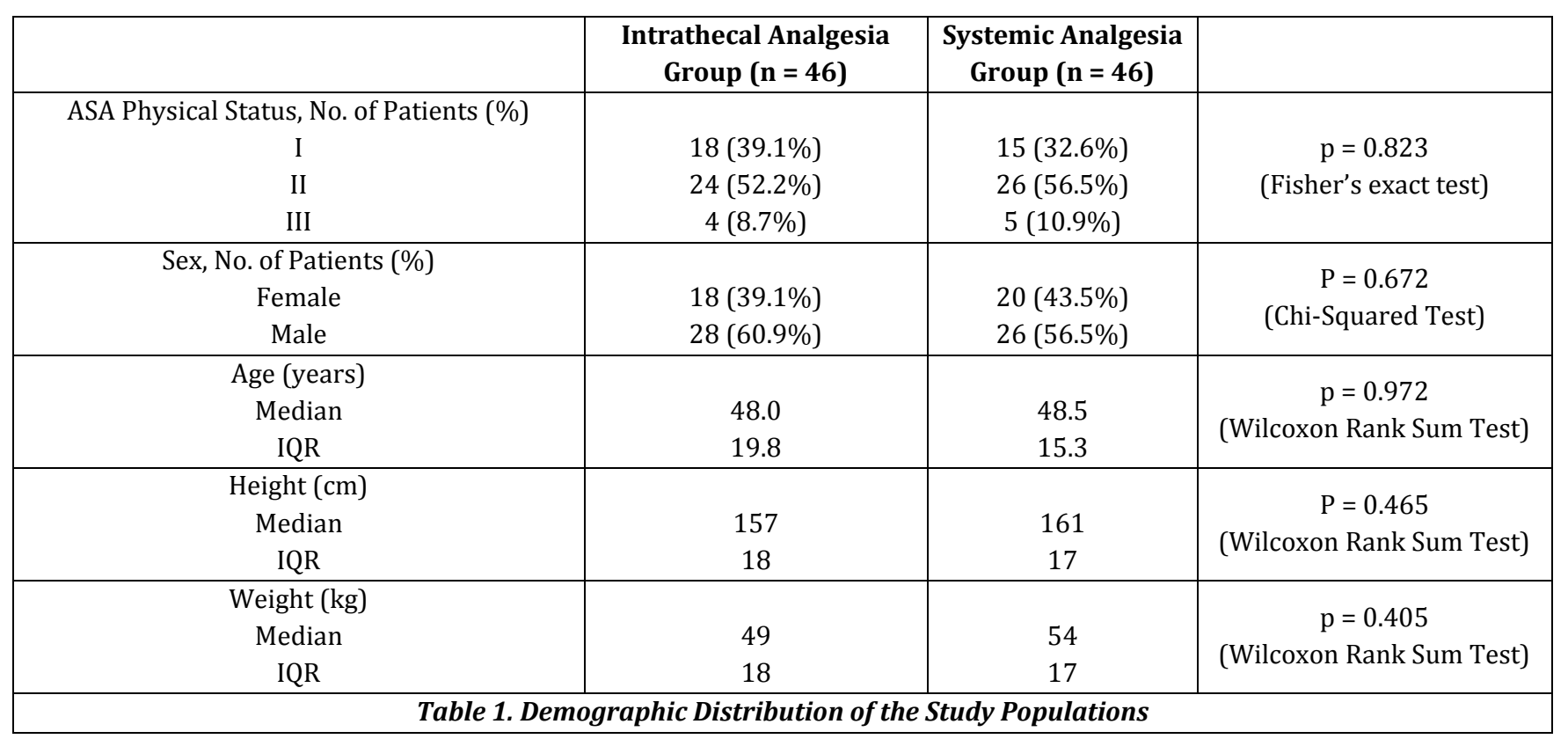




\begin{tabular}{|c|c|c|c|}
\hline & $\begin{array}{c}\text { Intrathecal Analgesia } \\
\text { Group (n = 46) }\end{array}$ & $\begin{array}{c}\text { Systemic Analgesia } \\
\text { Group (n = 46) }\end{array}$ & $\begin{array}{c}\text { Wilcoxon } \\
\text { Rank Sum Test }\end{array}$ \\
\hline $\begin{array}{c}\text { Duration of Surgery (minutes) } \\
\text { Median, (IQR) }\end{array}$ & $180,(88)$ & $165,(60)$ & $\mathrm{p}=0.763$ \\
\hline $\begin{array}{c}\text { Pain intensity (NRS) at 30 minutes after tracheal } \\
\text { extubation Median, (IQR) }\end{array}$ & $0,(0)$ & $2,(2)$ & $\mathrm{p}=0.0000006$ \\
\hline $\begin{array}{c}\text { Pain intensity (NRS) at 12 hours after tracheal } \\
\text { extubation Median, (IQR) }\end{array}$ & $1,(1)$ & $3,(1)$ & $\mathrm{p}=0.0000015$ \\
\hline $\begin{array}{c}\text { Pain intensity (NRS) at 24 hours after tracheal } \\
\text { extubation Median, (IQR) }\end{array}$ & $2,(2)$ & $3,(1)$ & $\mathrm{p}=0.0002$ \\
\hline $\begin{array}{c}\text { Total Morphine consumption (mg) in the first 24 } \\
\text { hours period after extubation Median, (IQR) }\end{array}$ & $10,(10)$ & $40,(10)$ & $\mathrm{p}=0.673$ \\
\hline $\begin{array}{c}\text { OASS Score at 30 minutes after tracheal extubation } \\
\text { Median, (IQR) }\end{array}$ & $4,(2)$ & $4,(2)$ & $\mathrm{p}=0.087$ \\
\hline $\begin{array}{c}\text { OASS Score at 12 hours after tracheal extubation } \\
\text { Median, (IQR) }\end{array}$ & $5,(2)$ & $5,(1)$ & $\mathrm{p}=0.537$ \\
\hline $\begin{array}{c}\text { OASS Score at 24 hours after tracheal extubation } \\
\text { Median, (IQR) }\end{array}$ & $4,(1)$ & $12,(0)$ & $\mathrm{p}=0.114$ \\
\hline $\begin{array}{c}\text { Total Mephentermine consumption (mg) in the } \\
\text { intraoperative period Median, (IQR) }\end{array}$ & $18,(6)$ & $1650,(800)$ & $\mathrm{p}=0.876$ \\
\hline $\begin{array}{c}\text { Urine output (mL) in the first 24 hours following } \\
\text { induction of anaesthesia Median, (IQR) }\end{array}$ & $1600,(1200)$ & Table 2. Comparison of Outcome Parameters of the Study Populations \\
\hline \multicolumn{2}{|c|}{}
\end{tabular}

\begin{tabular}{|c|c|c|c|c|c|c|}
\hline & $\begin{array}{c}\text { Intrathecal } \\
\text { Analgesia Group } \\
(\mathrm{n}=46)\end{array}$ & $\begin{array}{c}\text { Systemic } \\
\text { Analgesia Group } \\
(\mathrm{n}=46)\end{array}$ & $\begin{array}{c}\text { Chi-Squared Test } \\
\text { (Fisher's Exact } \\
\text { Test) }\end{array}$ & ARR & NNT & RRR \\
\hline $\begin{array}{l}\text { At } 30 \text { minutes } \\
\text { after tracheal } \\
\text { extubation }\end{array}$ & 0 & 0 & - & - & - & - \\
\hline $\begin{array}{c}\text { At } 12 \text { hours after } \\
\text { tracheal } \\
\text { extubation }\end{array}$ & $\begin{array}{c}1 \\
(2.17 \%)\end{array}$ & $\begin{array}{c}15 \\
(32.61 \%)\end{array}$ & $\begin{array}{l}p=0.00012 \\
(p=0.0002)\end{array}$ & $\begin{array}{c}0.3043 \\
\text { (95\% CI: } 0.1554 \\
\text { to } 0.4497)\end{array}$ & $\begin{array}{c}3 \\
\text { (95\% CI: } \\
6 \text { to } 2 \text { ) }\end{array}$ & $\begin{array}{c}0.9333 \\
\text { (95\% CI: } 0.5159 \\
\text { to } 0.9908)\end{array}$ \\
\hline $\begin{array}{c}\text { At } 24 \text { hours after } \\
\text { tracheal } \\
\text { extubation }\end{array}$ & $\begin{array}{c}3 \\
(6.52 \%)\end{array}$ & $\begin{array}{c}18 \\
(39.13 \%)\end{array}$ & $\begin{array}{c}p=0.0002 \\
(p=0.0003)\end{array}$ & $\begin{array}{c}0.3261 \\
\text { (95\% CI: } 0.1579 \\
\text { to } 0.4764)\end{array}$ & $\begin{array}{c}3 \\
\text { (95\% CI: } \\
6 \text { to } 2 \text { ) }\end{array}$ & $\begin{array}{c}0.8333 \\
\text { (95\% CI: } 0.4726 \\
\text { to } 0.9473)\end{array}$ \\
\hline
\end{tabular}

Absolute Risk Reduction; NNT = Number Needed to Treat; RRR = Relative Risk Reduction

\begin{tabular}{|c|c|c|c|}
\hline & $\begin{array}{l}\text { Intrathecal Analgesia } \\
\text { Group }(n=46)\end{array}$ & $\begin{array}{l}\text { Systemic Analgesia } \\
\text { Group }(n=46)\end{array}$ & \\
\hline $\begin{array}{l}\text { Nausea and Vomiting } \\
\text { No } \\
\text { Yes }\end{array}$ & $\begin{array}{l}30(65.2 \%) \\
16(34.8 \%)\end{array}$ & $\begin{array}{l}28(60.9 \%) \\
18(39.1 \%)\end{array}$ & $\begin{array}{c}\mathrm{p}=0.666 \\
\text { (Chi-squared test) }\end{array}$ \\
\hline $\begin{array}{l}\text { Mild Pruritus } \\
\text { No } \\
\text { Yes }\end{array}$ & $\begin{array}{c}6(13.0 \%) \\
40(87.0 \%)\end{array}$ & $\begin{array}{c}44(95.7 \%) \\
2(4.3 \%)\end{array}$ & $\begin{array}{c}\mathrm{p}=0.00000025 \\
\text { (Fisher's exact test) }\end{array}$ \\
\hline $\begin{array}{c}\text { Severe Pruritus requiring treatment } \\
\text { No } \\
\text { Yes }\end{array}$ & $\begin{array}{c}43(93.5 \%) \\
3(6.5 \%)\end{array}$ & $\begin{array}{l}46(100 \%) \\
0(0 \%)\end{array}$ & $\begin{array}{c}\mathrm{p}=0.242 \\
\text { (Fisher's exact test) }\end{array}$ \\
\hline $\begin{array}{c}\text { Michigan Awareness Classification Instrument Class } \\
0 \\
1 \\
2\end{array}$ & $\begin{array}{c}43(93.5 \%) \\
2(4.3 \%) \\
1(2.2 \%)\end{array}$ & $\begin{array}{l}40(87.0 \%) \\
4(8.7 \%) \\
2(4.3 \%)\end{array}$ & $\begin{array}{c}\mathrm{p}=0.571 \\
\text { (Fisher's exact test) }\end{array}$ \\
\hline \multicolumn{4}{|c|}{ Table 4. Comparison of Outcome Parameters of the Study Populations } \\
\hline
\end{tabular}




\section{DISCUSSION}

Dolin et al (2002) in a systematic review examined the evidence from published data concerning the incidence of moderate-severe and of severe pain after major surgery with three analgesic techniques; intramuscular (IM) analgesia, patient controlled analgesia (PCA) and epidural analgesia. Over 800 original papers and reviews were identified. Of these 212 papers fulfilled the inclusion criteria, but only 165 provided usable data on pain intensity and pain relief. Pooled data on pain scores obtained from these studies, which represent the experience of a total of nearly 20000 patients, form the basis of the review. Different pain measurement tools provided comparable data. When considering a mixture of three analgesic techniques, the overall mean $(95 \% \mathrm{Cl})$ incidence of moderate-severe pain and of severe pain was 29.7 (26.4 - 33.0) \% and 10.9 (8.4 - 13.4) \%, respectively. The overall mean $(95 \% \mathrm{Cl})$ incidence of poor pain relief and of fair-to-poor pain relief was $3.5(2.4-4.6) \%$ and 19.4 (16.4 22.3) \%, respectively. For IM analgesia, the incidence of moderate-severe pain was $67.2(58.1$ - 76.2) \% and that of severe pain was 29.1 (18.8 - 39.4) \%. For PCA, the incidence of moderate-severe pain was 35.8 (31.4 - 40.2) \% and that of severe pain was 10.4 (8.0 - 12.8) \%. For epidural analgesia, the incidence of moderate-severe pain was 20.9 (17.8 - 24.0) $\%$ and that of severe pain was $7.8(6.1-9.5) \% .{ }^{[1]}$

Gwirtz et al (1999) prospectively evaluated 5969 adult patients who received intrathecal opioid analgesia (ITOA) to manage postsurgical pain. To assess the efficacy of the analgesic technique and the incidence of complications, daily quality assurance data were collected on the first postoperative day and tabulated for who had received ITOA for major urologic, orthopaedic, general/vascular, thoracic and non-obstetrical gynaecologic surgery. A scale of $1-10$ was used to quantify each patient's satisfaction with analgesia. The incidence of side effects, complications and naloxone usage was also recorded and tabulated. The mean satisfaction score using a 10-point numeric rating scale was 8.51 with a score of 1 connoting "complete dissatisfaction" and 10 connoting "complete satisfaction." Side effects were minor and easily managed. Pruritus was the most common (37\%). Respiratory depression was the least common (3\%), easily detected by nursing observation, never life-threatening and always responsive to treatment with naloxone. Postdural puncture headaches were rare $(0.54 \%)$. There were no deaths, nerve injuries, central nervous system infections or naloxone related complications.[2]

Wongyingsinn et al (2012) have shown in a randomised controlled trial that spinal analgesia with morphine combined with bupivacaine for laparoscopic colonic resection provide better analgesia and decreases postoperative opioid requirement than those treated with intravenous opioid PCA.[8]

Our study is novel as we compared the safety and efficacy of intrathecal analgesia with morphine and bupivacaine combined with relaxant general anaesthesia and controlled ventilation, without use of anaesthesia workstation, anaesthetic gases and inhalation agents as opposed to a traditional anaesthetic protocol for major abdominal surgery. The anaesthetic and perioperative protocol in our experimental group simulated that of resource poor environment without availability of major expensive resources like anaesthesia workstation, anaesthetic gases and inhalation agents.

Our experimental protocol conferred significant absolute risk reduction in the failure of adequate postoperative analgesia at 12 hours and 24 hours compared to the control group using traditional protocol (Table 3). The number needed to treat for such absolute risk reduction was three.

Patients in the experimental group received intravenous midazolam for prevention of awareness under anaesthesia, as opposed to isoflurane in the control group. We have observed no significant differences in the postoperative OASS scores as well as awareness measured using Michigan Awareness Classification Instrument Class (based on a modified Brice Questionnaire). However, these were not the primary end points of this study. Neither this study was powered to detect statistically significant differences regarding the OASS score and Michigan Awareness Classification Instrument Class.

We have established that our experimental anaesthetic protocol is not only safe and less resource intensive, it has significantly lower incidence of failure of adequate postoperative analgesia in the first $24 \mathrm{~h}$ as compared to a traditional anaesthetic protocol for major abdominal surgery.

\section{CONCLUSION}

Intrathecal analgesia with morphine-bupivacaine combined with relaxant general anaesthesia and intravenous midazolam in a simulated resource poor setting (without anaesthesia workstation, nitrous oxide and volatile agent) is safe for a wide range of major abdominal surgery. This protocol is superior to traditional general anaesthesia protocol for major abdominal surgery using systemic multimodal analgesia, anaesthesia workstation, nitrous oxide and isoflurane in terms of failure rate of adequate analgesia in the first 24 hours postoperative period.

\section{REFERENCES}

[1] Dolin SJ, Cashman JN, Bland JM. Effectiveness of acute postoperative pain management: I. Evidence from published data. Br J Anaesth 2002;89(3):409-23.

[2] Gwirtz KH, Young JV, Byers RS, et al. The safety and efficacy of intrathecal opioid analgesia for acute postoperative pain: seven years' experience with 5969 surgical patients at Indiana University hospital. Anesth Analg 1999;88(3):599-604.

[3] Meylan N, Elia N, Lysakowski C, et al. Benefit and risk of intrathecal morphine without local anaesthetic in patients undergoing major surgery: meta-analysis of randomized trials. Br J Anaesth 2009;102(2):156-67.

[4] Zisman E, Shenderey A, Ammar R, et al. The effects of intrathecal morphine on patients undergoing minimally invasive direct coronary artery bypass surgery. J Cardiothorac Vasc Anesth 2005;19(1):40-3.

[5] Fléron $M H$, Weiskopf $R B$, Bertrand $M$, et al. $A$ comparison of intrathecal opioid and intravenous analgesia for the incidence of cardiovascular, respiratory and renal complications after abdominal aortic surgery. Anesth Analg 2003;97(1):2-12.

[6] Girgin NK, Gurbet A, Turker G, et al. Intrathecal morphine in anaesthesia for caesarean delivery: doseresponse relationship for combinations of low-dose intrathecal morphine and spinal bupivacaine. J Clin Anesth 2008;20(3):180-5. 
[7] Gehling MH, Luesebrink T, Kulka PJ, et al. The effective duration of analgesia after intrathecal morphine in patients without additional opioid analgesia: a randomized double-blind multicentre study on orthopaedic patients. Eur J Anaesthesiol 2009;26(8):683-8.

[8] Wongyingsinn M, Baldini G, Stein B, et al. Spinal analgesia for laparoscopic colonic resection using an enhanced recovery after surgery programme: better analgesia, but no benefits on postoperative recovery: a randomized controlled trial. $\mathrm{Br} \mathrm{J}$ Anaesth 2012;108(5):850-6.

[9] Chernik DA, Gillings D, Laine $\mathrm{H}$, et al. Validity and reliability of the Observer's Assessment of Alertness/Sedation Scale: study with intravenous midazolam. J Clin Psychopharmacol 1990;10(4):24451 .
[10] Mashour GA, Tremper KK, Avidan MS. Protocol for the "Michigan Awareness Control Study": a prospective, randomized, controlled trial comparing electronic alerts based on bispectral index monitoring or minimum alveolar concentration for the prevention of intraoperative awareness. BMC Anesthesiol 2009;9:7.

[11] Herbert R, George Institute, New South Wales, Australia. www.pedro.org.au/wp-content/uploads/ CIcalculator.xls. (Accessed August 27, 2017). 